Check for updates

Cite this: RSC Adv., 2018, 8, 11862

Received 21st December 2017

Accepted 1st March 2018

DOI: 10.1039/c7ra13550e

rsc.li/rsc-advances

\section{Highly flexible touch screen panel fabricated with silver-inserted transparent ITO triple-layer structures}

\begin{abstract}
Chia-Ching Wu (D)*
A flexible and transparent amorphous-indium tin oxide/silver/crystalline-indium tin oxide (a-ITO/Ag/c-ITO) triple-layer structure was prepared as an electrode for capacitive-type touch screen panels (TSPs). A very thin metal film of silver (Ag) was inserted between two ITO layers, and the triple-layer structures were deposited on a colorless polyimide (CPI) substrate by a sputtering method. It was found that the tunable electrical and optical properties of the a-ITO/Ag/c-ITO triple-layer structures were critically affected by the thickness of the inserted Ag layer. The optimized flexible a-ITO/Ag/c-ITO triple-layer structure has low sheet resistance, high optical transmittance, and low surface roughness. In addition, during the 30000 bending cycles, the resistance change $(\Delta R)$ of the flexible a-ITO/Ag/c-ITO triple-layer structure was $4.12 \%$. For environmental reliability, the $\Delta R$ values of the flexible a-ITO/Ag/c-ITO triple-layer structure were $2.86 \%$ and $0.96 \%$ at the environmental temperature of $80{ }^{\circ} \mathrm{C}-50 \%$ and $-40{ }^{\circ} \mathrm{C}$, respectively. The above results indicate that the a-ITO/Ag/c-ITO triple-layer structure can be utilized to construct a promising TCO electrode. Finally, flexible and foldable capacitive-type TSPs were fabricated with multiple touch points using the a-ITO/Ag/c-ITO triple-layer electrode.
\end{abstract}

\section{Introduction}

Transparent conducting oxide (TCO) is essential for photosensitive electronic devices such as thin film solar cells, ${ }^{1}$ flat panel displays, ${ }^{2}$ touch screens, ${ }^{3}$ and organic light-emitting diodes (OLEDs). ${ }^{4}$ There are at least two important factors, including the resistivity and transmittance, that are used to evaluate the performance of a TCO thin film. Low sheet resistance and high transmittance are required especially when TCO is used as an electrode material. Due to the great advance of experimental exploration, a large variety of TCO thin films, including traditional metal oxides, ${ }^{5,6}$ graphene, ${ }^{7}$ carbon nanotubes,${ }^{8}$ and metal nanostructures, ${ }^{9-15}$ have been successfully fabricated and widely applied in optoelectronic devices. Indium tin oxide (ITO) is the best choice for transparent conducting (TCO) films because of its low resistivity $\left(<10^{-4} \Omega \mathrm{cm}\right)$ and high transmittance $(>90 \%)$.

Touch screen panels (TSPs) have been considered as one of the key components in mobile communication devices. The capacitive-type TSPs are now being used instead of conventional resistive-type TSPs due to their capacity for multi-touch function and multitasking. Recently, flexible capacitive-type TSPs have been extensively investigated for application in flexible OLED displays. In these, the TCO electrode plays an important role in determining the performance of the flexible TSPs. The

Department of Applied Science, National Taitung University, Taitung, Taiwan, Republic of China. E-mail: 9113718@gmail.com; Fax: +886-089-517900; Tel: +886089-518108 flexible TCO electrode should have mechanical robustness against substrate bending without resultant changes in its optical and electrical properties. In previous research, amorphous ITO thin film were widely employed as a flexible TCO material in flexible optoelectronic devices due to their high conductivity and transparency in the visible spectral range. ${ }^{\mathbf{1 6}}$ However, cracks that easily form in the brittle amorphous ITO films have been considered as a critical drawback in flexible devices. ${ }^{17}$ In addition, crystallized ITO films showed a higher transmittance and lower resistivity than amorphous films. ${ }^{18,19}$

In order to eliminate the critical drawbacks of the ITO films, ITO sandwiching of thin metal films has been extensively investigated. ${ }^{20,21}$ Compared with single-layer ITO films, the ITO/ metal/ITO triple-layer structures can effectively suppress the reflection from the metal layer in the visible range and yield better electrical conductivity, and therefore, this area of research has generated interest. The metal layer of TCO/metal/ TCO multilayer structures is often comprised of gold $(\mathrm{Au})$, silver $(\mathrm{Ag})$, copper $(\mathrm{Cu})$, or molybdenum (Mo). Triple-layer structures such as ITO/metal/ITO, ${ }^{22,23} \mathrm{ZnO} / \mathrm{Cu} / \mathrm{ZnO},{ }^{24} \mathrm{IZO} / \mathrm{Au} / \mathrm{IZO},{ }^{25} \mathrm{ZnO} /$ $\mathrm{Ag} / \mathrm{ZnO},{ }^{26} \mathrm{AZO} / \mathrm{Ag} / \mathrm{AZO},{ }^{27} \mathrm{NTO} / \mathrm{Ag} / \mathrm{NTO},{ }^{28}$ and $\mathrm{ZTO} / \mathrm{Ag} / \mathrm{ZTO}^{29}$ have been reported.

In this research, we studied the structural, electrical, and optical properties of the ITO/metal/ITO triple-layer structures, which were sputtered on a colorless polyimide (CPI) substrate. The transmittance, coefficient of thermal expansion, and hardness of the CPI were outstanding for plastic material as compared to that of polyimide (PI), polyethylene terephthalate 
(PET), polyethylene naphthalate (PEN), and polyester (PES). The effects of the thin metal film thickness of the ITO/metal/ITO triple-layer structures were investigated. Under optimized conditions, we fabricated a flexible capacitive-type TSP using the ITO/metal/ITO triple-layer structure. The capacitive-type TSPs have been substituted for conventional resistive-type TSPs due to their capacity for multi-touch function and multitasking. The multi-touch function of TSPs is critically dependent on the resistance and optical transparency of the touch sensor electrodes. Thus, this study can provide a practical solution to the existing problems characterizing flexible TCO materials and their TSP application.

\section{Experimental detail}

\subsection{Fabrication of the ITO/Ag/ITO triple-layer structures}

In previous reports, the higher transmittance of the TCO-AgTCO structure compared with that of the TCO-Au-TCO structure was explained by $\mathrm{Ag}$ having a lower absorption than $\mathrm{Au}$ in the visible region of $400-700 \mathrm{~nm}$ (5\% versus $8 \%)^{30-32}$ In this investigation, a thin metal film of $\mathrm{Ag}$ was inserted between two flexible ITO thin films to form a triple-layer structure. The ITO/Ag/ITO triple-layer structures were prepared using a radio frequency (RF) magnetron sputtering method on a colorless polyimide (CPI) substrate under optimized top and bottom ITO thin film deposition conditions as a function of Ag thickness. The thickness of the CPI substrate was $10 \mu \mathrm{m}$. The distance between the CPI substrate and the target was approximately $5 \mathrm{~cm}$, and the substrate holder rotated so that a uniform film morphology was achieved during deposition. The sputtering deposition parameters of the ITO film and $\mathrm{Ag}$ thin metal film were base pressure of $3 \times 10^{-6}$ torr, pure Ar flow rate of $50 \mathrm{sccm}$, and working pressure of $5 \times 10^{-3}$ torr. The RF power of the ITO thin film was $80 \mathrm{~W}$, and for the Ag thin metal film, was $50 \mathrm{~W}$. The thicknesses of the Ag thin metal film were $1.5 \mathrm{~nm}, 2.9 \mathrm{~nm}$, $5.7 \mathrm{~nm}, 8.5 \mathrm{~nm}, 11.4 \mathrm{~nm}, 14.2 \mathrm{~nm}$, and $17.2 \mathrm{~nm}$ with deposition times of $10 \mathrm{~s}, 20 \mathrm{~s}, 40 \mathrm{~s}, 60 \mathrm{~s}, 80 \mathrm{~s}, 100 \mathrm{~s}$ and $120 \mathrm{~s}$, respectively. The detailed deposition parameters of the ITO thin film and the Ag thin metal film are shown in Table 1. Finally, the ITO/Ag/ITO triple-layer structures were annealed in the air for $1 \mathrm{~h}$ at $300^{\circ} \mathrm{C}$.

\subsection{Analysis of the ITO/Ag/ITO triple-layer structures}

Using a UV-Vis spectrometer, the optical transmittances of the ITO/Ag/ITO triple-layer structures were measured at wavelengths

Table 1 Deposition parameters of the ITO/Ag/ITO triple-layer structures

\begin{tabular}{llll}
\hline Parameter & Condition & & \\
\hline Target & Bottom ITO & Ag & Top ITO \\
Substrate & CPI & & \\
Working pressure (torr) & $5 \times 10^{-3}$ & & \\
Radio frequency power $(\mathrm{W})$ & 80 & 50 & 80 \\
Substrate temperature $\left({ }^{\circ} \mathrm{C}\right)$ & 160 & $\mathrm{RT}$ & $\mathrm{RT}$ \\
Deposition time (s) & 120 & 10 to 120 & 120 \\
Argon concentration $(\mathrm{sccm})$ & 50 & &
\end{tabular}

between 300 and $1100 \mathrm{~nm}$. The sheet resistances of the ITO/Ag/ ITO triple-layer structures were measured using four probe methods. The microstructure of the optimized ITO/Ag/ITO triplelayer structures was examined using high resolution transmission electron microscopy (HR-TEM). The TEM images were obtained from a cross-sectional HR-TEM specimen prepared via focus ion beam (FIB) milling. The resistivity $(\rho)$, carrier concentration $(n)$, and mobility $(\mu)$ of the ITO/Ag/ITO triple-layer structures were obtained from Hall-effect measurements. The mechanical and temperature reliability of the $\mathrm{ITO} / \mathrm{Ag} / \mathrm{ITO}$ triplelayer structures were investigated using a computer-controlled bending test machine and environmental reliability test system, respectively.

To investigate the feasibility of the ITO/Ag/ITO triple-layer structure as an electrode for capacitive-type TSPs, we fabricated a single-sided ITO (SITO) structure of the TSPs using an ITO/Ag/ITO electrode. The ITO/Ag/ITO electrode was directly deposited, and then patterned by a conventional photolithography and wet-etching process. The flexible printed circuit board (FPCB) bonding with the metal pattern progressed using anisotropic conductive film (ACF), and the FPCB was connected to the touch circuit controller. The touch circuit was designed by HannsTouch Solution Incorporated, in which the touch integrated circuit (IC) was purchased from Synaptics. After the touch sensor was connected to the touch circuit controller, firmware tuning was required. Finally, the plastic cover lens was attached to the touch sensor, and then the fabrication of the TSP was completed.

\section{Results and discussion}

In this investigation, a thin metal film of silver $(\mathrm{Ag})$ was inserted between flexible indium tin oxide (ITO) thin films to form ITO/ $\mathrm{Ag} / \mathrm{ITO}$ triple-layer structures. The transmittance spectra of the flexible ITO/Ag/ITO triple-layer structures as a function of $\mathrm{Ag}$ deposition time in wavelengths ranging from 300 to $1100 \mathrm{~nm}$ are shown in Fig. 1. In Fig. 1, the transmittance of the CPI

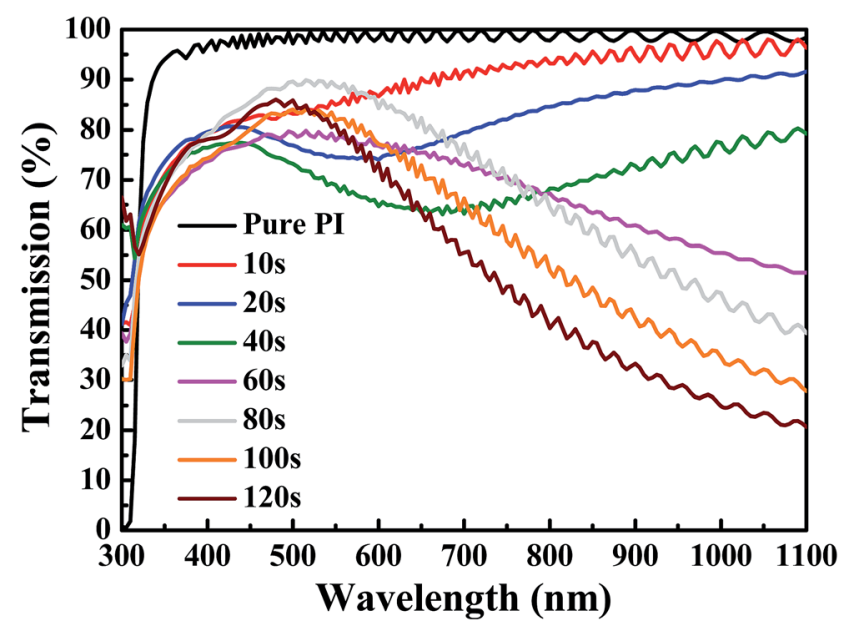

Fig. 1 UV-Vis spectra of the flexible ITO/Ag/ITO triple-layer structures as a function of $\mathrm{Ag}$ deposition time. 
substrate is $98.1 \%$ in the visible region and near-infrared region (NIR) without deposition of the flexible ITO/Ag/ITO triple-layer structures. At $\mathrm{Ag}$ deposition times of 10, 20, and $40 \mathrm{~s}$, the transmittance of the flexible ITO/Ag/ITO triple-layer structures shows a sharp increase in the near-ultraviolet (NUV), and then slightly decreases in the visible range, with a subsequent transmittance increase in the NIR. The flexible ITO/Ag/ITO triple-layer structures show a high transmittance in the visible region, with a subsequent slight decrease in the transmittance in the NIR as the $\mathrm{Ag}$ deposition time increases from 60 to $80 \mathrm{~s}$. Further increasing the Ag deposition time from 100 to $120 \mathrm{~s}$, the transmittance decreases again in the visible region and rapidly decreases in the NIR. It is obvious that the overall transmittance of the ITO/Ag/ITO triple-layer structures is highly influenced by the Ag deposition time. Fig. 2 shows a transmittance at $550 \mathrm{~nm}$ and average transmittance at 400-700 $\mathrm{nm}$ of the flexible ITO/Ag/ ITO triple-layer structures as a function of $\mathrm{Ag}$ deposition time. The transmittance at $550 \mathrm{~nm}$ was $85.3 \%, 74.5 \%, 69.2 \%, 77.9 \%$, $88.7 \%, 81.9 \%$, and $81.1 \%$ for $\mathrm{Ag}$ deposition times of 10, 20, 40, $60,80,100$, and $120 \mathrm{~s}$, respectively, as shown in Fig. 2. It was found that the transmittance of the flexible ITO/Ag/ITO triplelayer structures was critically influenced by the thickness of the inserted $\mathrm{Ag}$ layer. The increase in $\mathrm{Ag}$ deposition times from 10 to $40 \mathrm{~s}$ decreased the transmittance at $550 \mathrm{~nm}$ and average transmittance at $400-700 \mathrm{~nm}$ of the flexible ITO/Ag/ITO triplelayer structures due to severe scattering of light by the separate islands randomly distributed between the ITO layers. Because the $\mathrm{Ag}$ islands act as a scattering source of incident light, the flexible ITO/Ag/ITO triple-layer structures are revealed as a bluish color. However, as the $\mathrm{Ag}$ deposition time increased from $40-80 \mathrm{~s}$, the $\mathrm{Ag}$ islands coalesced to a continuous film that remarkably increased the transmittance and average transmittance in the same wavelength region. The inset in Fig. 2 shows surface SEM images of the ITO/Ag/ITO triple-layer structures that were deposited at the $40 \mathrm{~s}$ and $80 \mathrm{~s} \mathrm{Ag}$ deposition time.

When an optimum $\mathrm{Ag}$ thin metal film is embedded between two oxide layers, the resulting oxide/metal/oxide (OMO) triplelayer structure can suppress reflections from the $\mathrm{Ag}$ thin

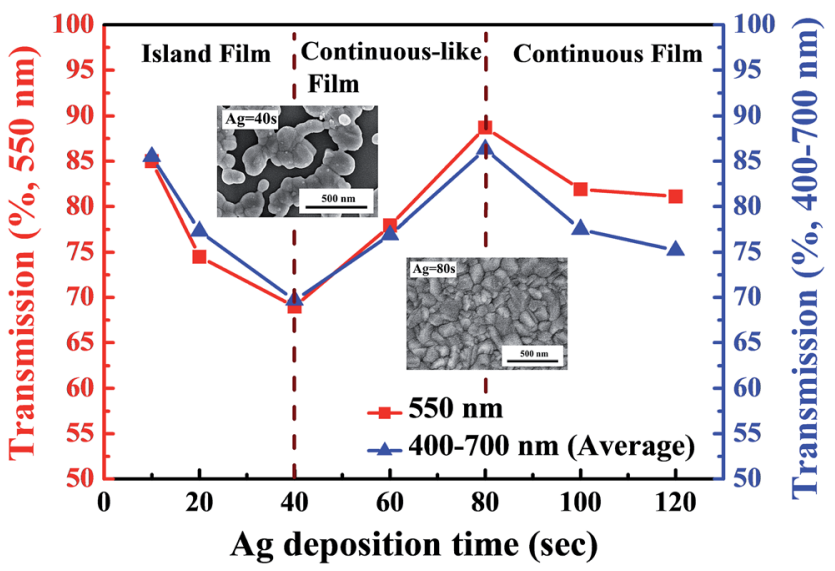

Fig. 2 Transmittance of the flexible ITO/Ag/ITO triple-layer structures as a function of Ag deposition time. metal film and results in a high transmittance in the visible wavelength region. ${ }^{33-36}$ Therefore, the maximum transmittance at $500 \mathrm{~nm}(88.7 \%)$ and $400-700 \mathrm{~nm}(86.2 \%)$ of the flexible ITO/ $\mathrm{Ag} / \mathrm{ITO}$ triple-layer structure was obtained with the $\mathrm{Ag}$ deposition time set to $80 \mathrm{~s}$. As the deposition times of the $\mathrm{Ag}$ are further increased, the transmittance and average transmittance of the flexible ITO/Ag/ITO triple-layer structures decreases due to the reflection on the thicker Ag metal film. In particular, the optical transmittance in the infrared wavelength region significantly decreased with increasing Ag thin metal film thickness, and this phenomenon was caused by the plasma resonance frequency effect. ${ }^{37}$ Consequently, by insertion of $\mathrm{Ag}$ thin metal films with different deposition times between the ITO, the effective plasma resonance frequency of the ITO/Ag/ITO triplelayer structure can be tuned accordingly.

Fig. 3 presents the resistivity $(\rho)$, carrier concentration $(n)$, and mobility $(\mu)$ of the flexible ITO/Ag/ITO triple-layer structures as a function of $\mathrm{Ag}$ deposition time. The resistivity of the flexible ITO/Ag/ITO triple-layer structures decreased from $9.12 \times 10^{-4}$ to $2.2 \times 10^{-5} \Omega \mathrm{cm}$ as the $\mathrm{Ag}$ deposition time increased from 10 to $120 \mathrm{~s}$. The decrease in resistivity is mainly due to the increases in both carrier concentration and mobility with the increase in $\mathrm{Ag}$ deposition time because the resistivity of the flexible ITO/Ag/ITO triple-layer structures is proportional to the reciprocal value of the product of the carrier concentration and the mobility. ${ }^{38}$

The carrier concentration increased from $2.65 \times 10^{20} \mathrm{~cm}^{-3}$ to $2.21 \times 10^{22} \mathrm{~cm}^{-3}$ as the $\mathrm{Ag}$ deposition time increased from 10 to $120 \mathrm{~s}$. As discussed by Alford Klöppel et al., the metal interlayer can act as an electron source for the oxide layer in the TCO/ metal/TCO structure. ${ }^{39}$ Therefore, the electron in the Ag layer can be easily injected into the ITO layer due to downward band bending at the contact by the difference in work functions between $\mathrm{Ag}\left(\varphi_{\mathrm{M}}=4.4 \mathrm{eV}\right)$ layer and ITO $\left(\varphi_{\mathrm{O}}=4.5-5.1 \mathrm{eV}\right)$ in the flexible ITO/Ag/ITO triple-layer structures. ${ }^{40,41}$ A schematic of the energy band diagram of the ITO and $\mathrm{Ag}$ prior to and after their contact is shown in Fig. 4(a) and (b), respectively. The mobility of the flexible ITO/Ag/ITO triple-layer structures

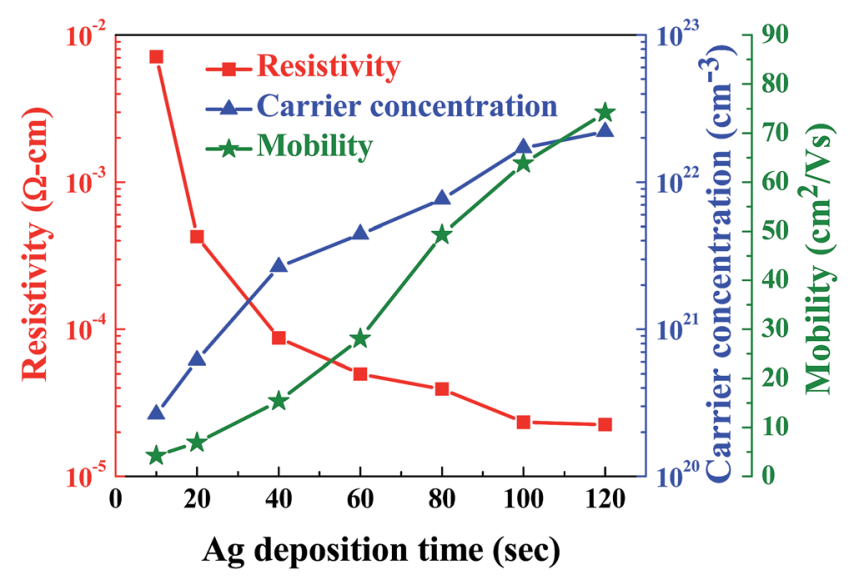

Fig. 3 The resistivity $(\rho)$, carrier concentration $(n)$, and mobility $(\mu)$ of the flexible ITO/Ag/ITO triple-layer structures as a function of $\mathrm{Ag}$ deposition time. 
(a)

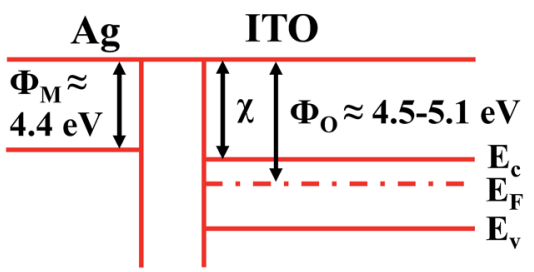

(b)

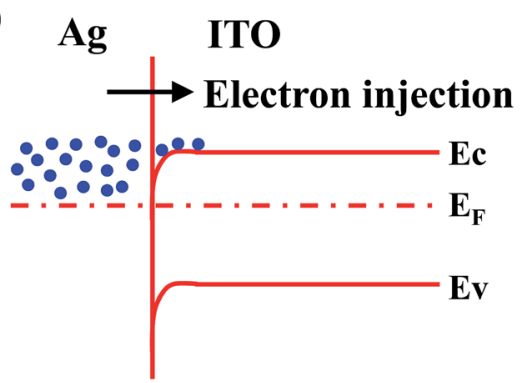

Fig. 4 Schematic diagrams of the energy band structures of the ITO and Ag: (a) before contact and (b) after contact.

increased from $4.2 \mathrm{~cm}^{2} \mathrm{~V}^{-1}$ to $74.3 \mathrm{~cm}^{2} \mathrm{~V}^{-1}$ as the $\mathrm{Ag}$ deposition time increased. The mobility increase is largely ascribed to the reduction of scattering at the interface regions between the metal and oxide layers, because the interface scattering is considered the main scattering mechanism in the flexible ITO/ Ag/ITO triple-layer structure. ${ }^{42}$

Fig. 5 shows the figure of merit (FOM) and sheet resistances of the flexible ITO/Ag/ITO triple-layer structures as a function of Ag deposition time. To obtain the best combination of high transmission and low resistivity, the FOM for the films was calculated using the Haacke equation, ${ }^{\mathbf{4 3}}$

$$
\varphi_{\mathrm{TC}}=\frac{T_{\mathrm{av}} 10}{R_{\mathrm{s}}}
$$

where $T_{\mathrm{av}}$ is the optical transmittance of the flexible ITO/Ag/ITO triple-layer structures at the $550 \mathrm{~nm}$ wavelength, and $R_{\mathrm{S}}$ is the sheet resistance. $T_{\mathrm{av}}$ can be estimated using eqn (2):

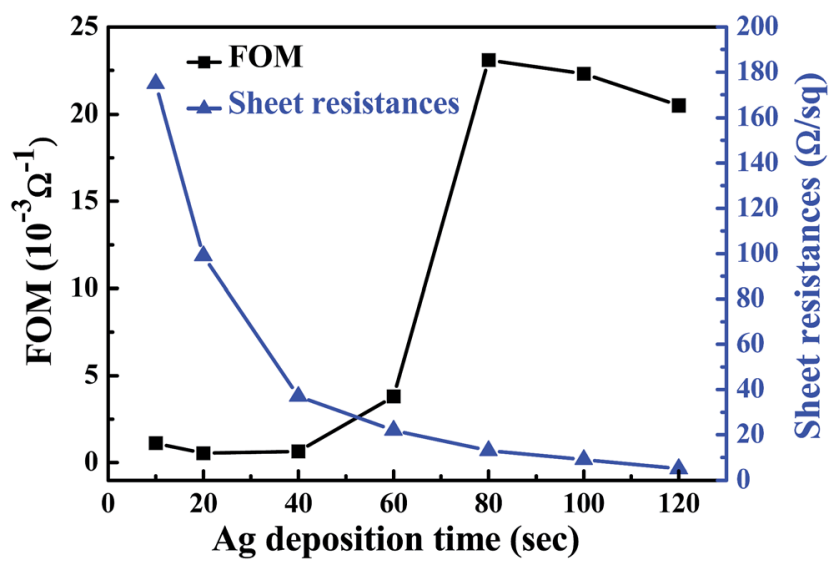

Fig. 5 FOM values and sheet resistances of the flexible ITO/Ag/ITO triple-layer structures as a function of Ag deposition time.

$$
T_{\mathrm{av}}=\frac{\int v(\lambda) T(\lambda) \mathrm{d}(\lambda)}{\int v(\lambda) \mathrm{d}(\lambda)}
$$

where $T(\lambda)$ is the transmittance, and $v(\lambda)$ is the photopic luminous efficiency function defining the standard observer for photometry. ${ }^{44}$ In Fig. 5, the maximum value of the FOM at 12.2 $\times 10^{-3} \Omega^{-1}$ can be observed at $80 \mathrm{~s} \mathrm{Ag}$ deposition time. The sheet resistance and transmittance at $550 \mathrm{~nm}$ were $13 \Omega \square^{-1}$ and $88.7 \%$, respectively. From the above results, high optical transmittance and low resistance of the flexible ITO/Ag/ITO triple-layer structure were obtained as the $\mathrm{Ag}$ deposition time was set to $80 \mathrm{~s}$ [abbreviation as ITO/Ag(80)/ITO].

To produce flexible ITO/Ag(80)/ITO triple-layer structures with higher transmittance, the different deposition parameters of the ITO thin film were investigated. Fig. 6 shows the transmittance spectra of the ITO/Ag(80)/ITO triple-layer structures at the deposition temperature of $160{ }^{\circ} \mathrm{C}$ for the bottom ITO thin film [abbreviated as ITO/Ag(80)/ITO(160)]. The transmittance at $550 \mathrm{~nm}$ and the average transmittance at $400-700 \mathrm{~nm}$ of the flexible ITO/Ag(80)/ITO(160) triple-layer structures were $91.4 \%$ and $87.5 \%$, respectively. The transmittance and the average transmittance of the flexible ITO/Ag(80)/ITO(160) triple-layer structure are higher than that of the flexible ITO/Ag(80)/ITO triple-layer structure. This was caused by the crystallized ITO thin film, which showed a higher transmittance and lower resistivity than the amorphous film. ${ }^{45,46}$ The sheet resistance of the flexible ITO/Ag(80)/ITO(160) triple-layer structure is $6.4 \Omega \square^{-1}$, and it is lower than that of the flexible ITO/Ag(80)/ ITO structure. Table 2 gives previously published values for the optical transmittance at $550 \mathrm{~nm}$, sheet resistance, and FOM values of transparent conductive films deposited on flexible substrates. It can be seen that the FOM value obtained in the present work is slightly higher than previously reported values.

The surface roughness value of the flexible ITO/Ag(80)/ ITO(160) triple-layer structure is very important for future

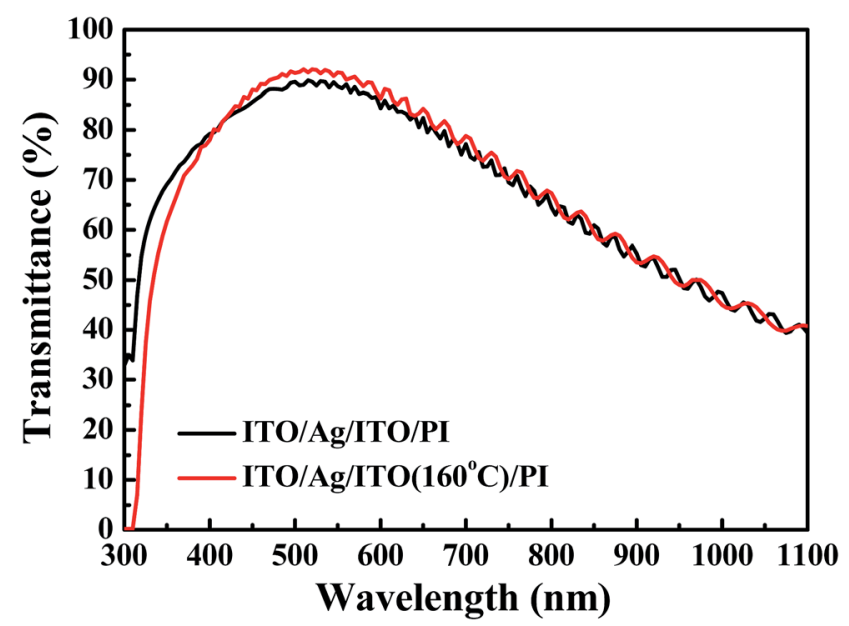

Fig. 6 UV-Vis spectra of the flexible ITO/Ag/ITO triple-layer structures with the deposition temperature at $160^{\circ} \mathrm{C}$ for the bottom ITO thin film at the $80 \mathrm{~s}$ Ag deposition time. 
Table 2 Maximum optical transmittance, minimum sheet resistance, and largest FOM of transparent conductive films reported in the literature

\begin{tabular}{|c|c|c|c|c|}
\hline 23.2 & 13 & 88.7 & ITO/Ag(80)/ITO/CPI & This work \\
\hline 118.5 & 1.16 & $91($ at $600 \mathrm{~nm})$ & ITO/PEI & 47 \\
\hline 7.87 & 19.7 & 83 & ITO/PI & 48 \\
\hline 6.66 & 8.93 & $82.4 \%$ & ITO/Ag/ITO/PET & 49 \\
\hline 11.6 & 30 & 90 & Graphene/PET & 52 \\
\hline 1.26 & 85 & 80 & SWNTs/PET & 53 \\
\hline
\end{tabular}

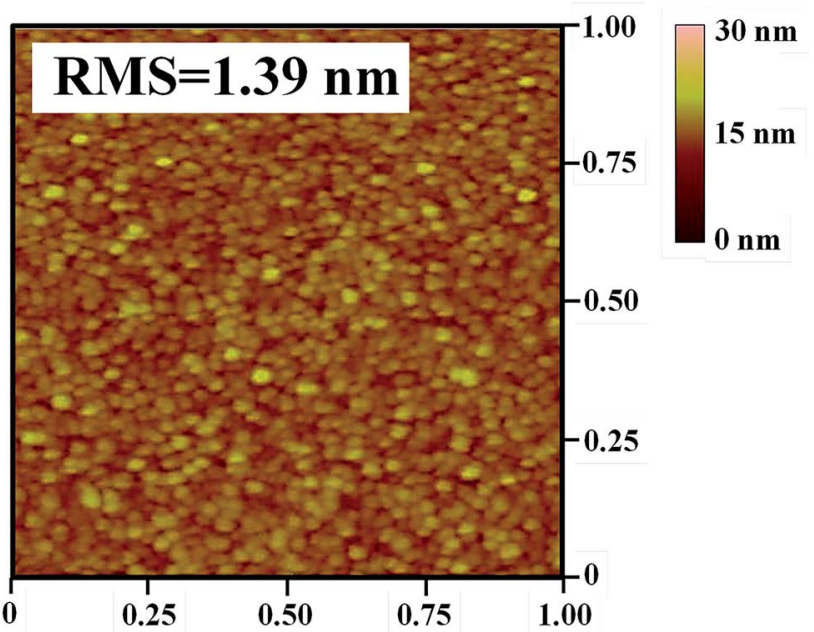

Fig. 7 AFM analysis of the flexible ITO/Ag/ITO triple-layer structure with deposition temperature at $160{ }^{\circ} \mathrm{C}$ for the bottom ITO thin film at the $80 \mathrm{~s}$ Ag deposition time. Scan area is $1 \mu \mathrm{m} \times 1 \mu \mathrm{m}$. applications. Fig. 7 shows the root mean square (RMS) roughness $(1 \mu \mathrm{m} \times 1 \mu \mathrm{m})$ of the flexible $\mathrm{ITO} / \mathrm{Ag}(80) / \mathrm{ITO}(160)$ triple-layer structure by atomic force microscopy (AFM). The RMS roughness of the flexible ITO/Ag(80)/ITO(160) triple-layer structure is $1.39 \mathrm{~nm}$. The RMS roughness of the top ITO layer is small and demonstrates the amorphous structure and decrease in the surface roughness. Therefore, the flexible ITO/ $\operatorname{Ag}(80) / \operatorname{ITO}(160)$ triple-layer structure is beneficial for further TSP device fabrication.

Fig. 8 shows cross-sectional transmission electron microscopy (TEM) images and the high-resolution TEM image of the ITO/Ag(80)/ITO(160) triple-layer structure. The gray and black images clearly show the triple-layer structure consisting of bottom ITO, Ag, and top ITO thin films without interfacial layers, as shown in Fig. 8(a). The thickness of the bottom ITO, $\mathrm{Ag}$, and top ITO thin films are $21.1 \mathrm{~nm}, 11.4 \mathrm{~nm}$, and $24.7 \mathrm{~nm}$, respectively. In the case of the ITO/Ag/ITO triple-layer structure in Fig. 8(b), the image shows that the bottom ITO thin film has a complete crystallization structure due to its deposition at the substrate temperature of $160^{\circ} \mathrm{C}$. It shows the bottom ITO and $\mathrm{Ag}$ layers without an interfacial layer. There are no interface reactions of interfacial oxide layers between the bottom ITO and
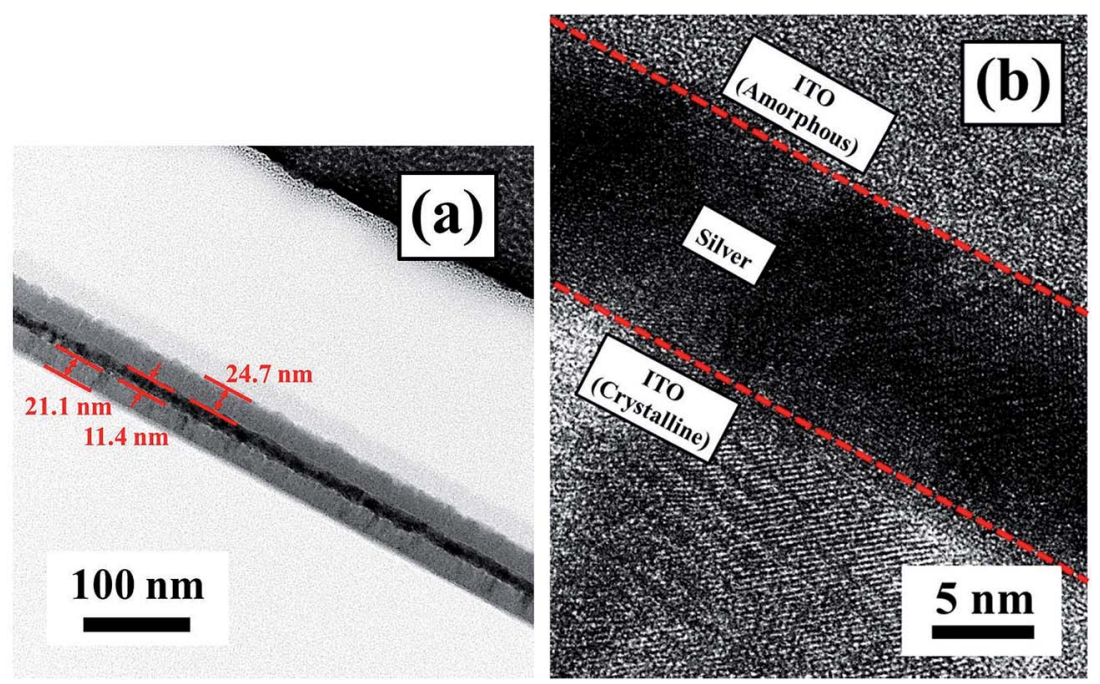

Fig. $8 \mathrm{TEM}$ image of the flexible ITO/Ag/ITO triple-layer structure with deposition temperature at $160^{\circ} \mathrm{C}$ for the bottom ITO thin film at the $80 \mathrm{~s}$ Ag deposition time. 
$\mathrm{Ag}$ layers due to the use of a continuous sputtering process without breaking the vacuum. In addition, before deposition of the Ag thin film, the $160{ }^{\circ} \mathrm{C}$ substrate temperature would be cooling down to room temperature. Finally, the amorphous structure of the top ITO thin film was obtained at deposition at room temperature. From the above results, it was evident that the crystallization of the ITO thin film enhances the transmittance of the ITO/Ag/ITO triple-layer structure, and the amorphous ITO thin film reduces the surface roughness.

To evaluate the mechanical reliability of the flexible ITO/ $\mathrm{Ag}(80) / \mathrm{ITO}(160)$ triple-layer structure for TSP application, we measured resistance changes $(\Delta R)$ in the flexible ITO/Ag(80)/ ITO(160) triple-layer structure during dynamic outer bending. Table 3 shows the bending reliability analysis of the flexible ITO/Ag(80)/ITO(160) triple-layer structure using a computercontrolled bending test machine with the bending radius set to $3 \mathrm{~mm}$. The critical bending radius of $3 \mathrm{~mm}$ was defined by the standard commercial test created by HannsTouch Solution Incorporated. The sheet resistance of the flexible ITO/Ag(80)/ ITO(160) triple-layer structure is $6.4 \Omega \square^{-1}$. Changes in the sheet resistance of the ITO/Ag(80)/ITO(160) triple-layer structure can be expressed as $\Delta R(\%)=\left(R_{1}-R_{0}\right) / R_{0}$, where $R_{0}$ is the initial resistance and $R_{1}$ is the measured resistance after bending. During the 15000 and 30000 bending cycles, the $\Delta R$ values of the flexible ITO/Ag(80)/ITO(160) triple-layer structure were $2.06 \%$ and $4.12 \%$, respectively. The dynamic outer bending of the flexible ITO/Ag(80)/ITO(160) triple-layer structure showed less change in sheet resistance after 30000 bending cycles, demonstrating good flexibility of the ITO/ $\mathrm{Ag}(80) / \mathrm{ITO}(160)$ triple-layer structure. This flexibility of the ITO/Ag(80)/ITO(160) triple-layer structure can be attributed to the ability of the metallic $\mathrm{Ag}$ thin interlayer between the ITO layers to withstand high strain, and even the local delamination of or crack formation in the top and bottom ITO thin films.

Environmental reliability is a very important test for commercialization of a material. The resistance change $(\Delta R)$ of the flexible ITO/Ag(80)/ITO(160) triple-layer structure was measured by using the environmental reliability test system. The high and low temperature are set to $80{ }^{\circ} \mathrm{C}-85 \%$ and $-40{ }^{\circ} \mathrm{C}$, respectively. The resistance of the flexible $\mathrm{ITO} / \mathrm{Ag}(80) / \mathrm{ITO}(160)$ triple-layer structure is $6.4 \Omega \square^{-1}$. During the environmental

Table 3 Bending reliability analysis and in situ measured resistance of the flexible ITO/Ag(80)/ITO(160) triple-layer structure

\begin{tabular}{lll}
\hline Bending radius & $\Delta R_{15 \mathrm{~K}}(\%)$ & $\Delta R_{30 \mathrm{~K}}(\%)$ \\
\hline $3 \mathrm{~mm}$ & $2.06 \%$ & $4.12 \%$
\end{tabular}

Table 4 Environmental reliability test and in situ measured resistance of the flexible ITO/Ag(80)/ITO(160) triple-layer structure

\begin{tabular}{lll}
\hline Temperature & $\Delta R_{120 \mathrm{~h}}(\%)$ & $\Delta R_{240 \mathrm{~h}}(\%)$ \\
\hline $80{ }^{\circ} \mathrm{C}-85 \%$ & $1.71 \%$ & $2.86 \%$ \\
$-40{ }^{\circ} \mathrm{C}$ & $0.96 \%$ & $0.96 \%$
\end{tabular}

Fig. 9 Optical microscope image of the patterned ITO/Ag/ITO triplelayer structure used to fabricate the flexible capacitive-type touch panels.

reliability test of $80{ }^{\circ} \mathrm{C}-85 \%$ and $-40{ }^{\circ} \mathrm{C}$, the $\Delta R$ of the flexible ITO/Ag(80)/ITO(160) triple-layer structure are $2.86 \%$ and $0.96 \%$ with $240 \mathrm{~h}$ as shown in Table 4, respectively. The flexible ITO/Ag(80)/ITO(160) triple-layer structure showed less change in resistance after $80{ }^{\circ} \mathrm{C}-85 \%$ and $-40{ }^{\circ} \mathrm{C}$ environmental reliability test, demonstrating the good reliability of the flexible ITO/Ag(80)/ITO(160) triple-layer structure.

Fig. 9 shows an optical microscopy (OM) image of the flexible ITO/Ag(80)/ITO(160) triple-layer structure of the fabricated capacitive-type TSPs. To fabricate the flexible capacitive-type TSPs, the ITO/Ag(80)/ITO(160) electrode was patterned by

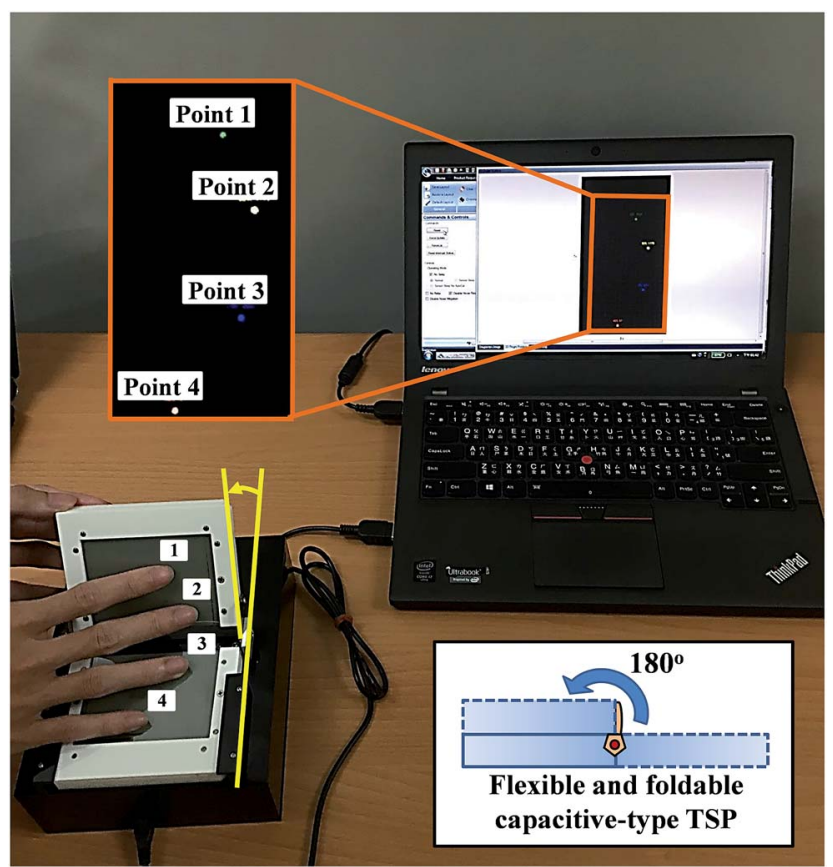

Fig. 10 Flexible and foldable capacitive-type TSP fabricated with the ITO/Ag/ITO electrode demonstrates multiple touch point function. 
a photolithography and wet etching process. The wet etchant is a phosphoric acid and nitric acid mixture. Using the well patterned ITO/Ag(80)/ITO(160) electrode, flexible capacitivetype TSPs were fabricated.

Fig. 10 shows the multiple touch point operation of a flexible capacitive-type TSP fabricated with the ITO/Ag/ITO electrode. The capacitive-type TSP was operated by exact sensing of $X-Y$ coordinates and characteristics of linearity. Operation of the flexible capacitive-type TSP with the ITO/Ag/ITO electrode indicates that the ITO/Ag/ITO triple-layer structure with low sheet resistance and high optical transmittance is a promising transparent electrode to substitute for the conventional ITO electrode. In this investigation, the maximum bending angle of the flexible capacitive-type TSP was $180^{\circ}$, as shown in Fig. 10 . To evaluate the practical usage of the flexible capacitive-type TSP, we lightly touched the TSP, and the four touch points were displayed on a notebook monitor, as shown in Fig. 10. This test verified that this flexible capacitive-type TSP can detect multiple touch points. We are currently in the process of fabricating small-sized capacitive-type TSPs for smartphones and applications combined with organic light emitting diode (OLED) displays.

\section{Conclusion}

An amorphous-indium tin oxide/silver/crystalline-indium tin oxide (a-ITO/Ag/c-ITO) triple-layer structure with low resistivity and high transmittance was studied as a substitute for conventional ITO electrodes for flexible capacitive-type touch screen panels (TSPs). Under optimized conditions, the a-ITO/ $\mathrm{Ag} / \mathrm{c}$-ITO (21.1 nm/11.4 nm/24.7 nm) triple-layer structure grown on a colorless polyimide (CPI) substrate exhibited a sheet resistance of $6.4 \Omega \square^{-1}$, an optical transmittance of $91.4 \%$, a surface roughness of $1.39 \mathrm{~nm}$, a resistance change $(\Delta R)$ with bending reliability of $4.12 \%$, and $\Delta R$ of $2.86 \%$ and $0.96 \%$ with environmental reliability of $80{ }^{\circ} \mathrm{C}-50 \% /-40{ }^{\circ} \mathrm{C}$, which is much better than conventional ITO thin film. In this investigation, the flexible and foldable capacitive-type TSP fabricated with a-ITO/ Ag/c-ITO electrode demonstrated multiple touch points function.

\section{Conflicts of interest}

There are no conflicts to declare.

\section{Acknowledgements}

The authors acknowledge the financial support of the Ministry of Science and Technology (105CE02, MOST 105-2221-E-244-013 and 106-2221-E-244-009). The authors also acknowledge HannsTouch Solution Incorporated for supplying the polyimide substrate and assisting with the fabrication of the flexible capacitive-type touch screen panel.

\section{References}

1 N. Formica, P. Mantilla-Perez, D. S. Ghosh, D. Janner, T. L. Chen, M. Huang, S. Garner, J. Martorell and
V. Pruneri, An Indium Tin Oxide-Free Polymer Solar Cell on Flexible Glass, ACS Appl. Mater. Interfaces, 2015, 7, 4541-4548.

2 P. S. Pa, Implementation of a Reuse Process for Liquid Crystal Displays Using an Eccentric-Form Tool, Int. J. Mol. Sci., 2009, 10, 4178-4186.

3 Y. H. Shin, C. K. Cho and H. K. Kim, Resistance and transparency tunable Ag-inserted transparent InZnO films for capacitive touch screen panels, Thin Solid Films, 2013, 548, 641-645.

4 S. W. Cho, J. A. Jeong, J. H. Bae, J. M. Moon, K. H. Choi, S. W. Jeong, N. J. Park, J. J. Kim, S. H. Lee, J. W. Kang, M. S. Yi and H. K. Kim, Highly flexible, transparent, and low resistance indium zinc oxide-Ag-indium zinc oxide multilayer anode on polyethylene terephthalate substrate for flexible organic light-emitting diodes, Thin Solid Films, 2008, 516, 7881-7885.

5 C. Singh and E. Panda, Variation of electrical properties in thickening Al-doped $\mathrm{ZnO}$ films: role of defect chemistry, RSC Adv., 2016, 6, 48910.

6 R. A. Maniyara, V. K. Mkhitaryan, T. L. Chen, D. S. Ghosh and V. Pruneri, An antireflection transparent conductor with ultralow optical loss $(<2 \%)$ and electrical resistance $(<6 \Omega$ $\mathrm{sq}^{-1}$ ), Nat. Commun., 2016, 7, 13771.

7 S. Bae, H. Kim, Y. Lee, X. F. Xu, J. S. Park, Y. Zheng, J. Balakrishnan, T. Lei, H. R. Kim, Y. I. Song, Y. J. Kim, K. S. Kim, B. Ozyilmaz, J. H. Ahn, B. H. Hong and S. Iijima, Nat. Nanotechnol., 2010, 5, 574.

8 N. Fukaya, D. Y. Kim, S. Kishimoto, S. Noda and Y. Ohno, One-Step Sub-10 $\mu \mathrm{m}$ Patterning of Carbon-Nanotube Thin Films for Transparent Conductor Applications, ACS Nano, 2014, 8, 3285.

9 Y. Ahn, Y. Jeong, D. Lee and Y. Lee, Copper NanowireGraphene Core-Shell Nanostructure for Highly Stable Transparent Conducting Electrodes, ACS Nano, 2015, 9, 3125-3133.

10 A. Kim, Y. Won, K. Woo, S. Jeong and J. Moon, All-SolutionProcessed Indium-Free Transparent Composite Electrodes based on Ag Nanowire and Metal Oxide for Thin-Film Solar Cells, Adv. Funct. Mater., 2014, 24, 2462-2471.

11 H. Moon, P. Won, J. Lee and S. H. Ko, Low-haze, annealingfree, very long $\mathrm{Ag}$ nanowire synthesis and its application in a flexible transparent touch panel, Nanotechnology, 2016, 27, 295201.

12 Y. D. Suh, S. Hong, J. Lee, H. Lee, S. Jung, J. Kwon, H. Moon, P. Won, J. Shin, J. Yeo and S. H. Ko, Random nanocrack, assisted metal nanowire bundled network fabrication for a highly flexible and transparent conductor, RSC Adv., 2016, 6, 57434-57440.

13 S. Hong, J. Yeo, J. Lee, H. Lee, P. Lee, S. S. Lee and S. H. Ko, Selective Laser Direct Patterning of Silver Nanowire Percolation Network Transparent Conductor for Capacitive Touch Panel, J. Nanosci. Nanotechnol., 2015, 15, 2317-2323.

14 P. Lee, J. Ham, J. Lee, S. Hong, S. Han, Y. D. Suh, S. E. Lee, J. b. Yeo, S. S. Lee, D. Lee and S. H. Ko, Highly Stretchable or Transparent Conductor Fabrication by a Hierarchical 
Multiscale Hybrid Nanocomposite, Adv. Funct. Mater., 2014, 24, 5671-5678.

15 J. Lee, P. Lee, H. B. Lee, S. Hong, I. Lee, J. Yeo, S. S. Lee, T. S. Kim, D. Lee and S. H. Ko, Room-Temperature Nanosoldering of a Very Long Metal Nanowire Network by Conducting-Polymer-Assisted Joining for a Flexible TouchPanel Application, Adv. Funct. Mater., 2013, 23, 4171-4176.

16 R. B. H. Tahar, T. Ban, Y. Ohya and Y. Takahashi, Tin doped indium oxide thin films: electrical properties, J. Appl. Phys., 1998, 83, 2631-2645.

17 Y. Leterrier, L. Medico, F. Demarco, J. A. E. Manson, U. Betz, M. F. Escola, M. K. Olsson and F. Atamny, Mechanical integrity of transparent conductive oxide films for flexible polymer-based displays, Thin Solid Films, 2004, 460, 156.

18 L. Kerkache, A. Layadi, E. Dogheche and D. Remiens, Physical properties of RF sputtered ITO thin films and annealing effect, J. Phys. D: Appl. Phys., 2006, 39, 18.

19 H. J. Choi, S. G. Yoon, J. H. Lee and J. Y. Lee, Crystallized Indium-Tin Oxide (ITO) Thin Films Grown at Low Temperature onto Flexible Polymer Substrates, ECS J. Solid State Sci. Technol., 2012, 1, Q106-Q109.

20 D. R. Sahu, S. Y. Lin and J. L. Huang, ZnO/Ag/ZnO multilayer films for the application of a very low resistance transparent electrode, Appl. Surf. Sci., 2006, 252, 7509-7514.

21 D. R. Sahu and J. L. Huang, High quality transparent conductive $\mathrm{ZnO} / \mathrm{Ag} / \mathrm{ZnO}$ multilayer films deposited at room temperature, Thin Solid Films, 2006, 515, 876.

22 D. Kim, Low temperature deposition of transparent conducting ITO/Au/ITO films by reactive magnetron sputtering, Appl. Surf. Sci., 2010, 256, 1774-1777.

23 J. A. Jeong, Y. Park and H. K. Kim, Comparison of electrical, optical, structural, and interface properties of IZO-Ag-IZO and IZO-Au-IZO multilayer electrodes for organic photovoltaics, J. Appl. Phys., 2010, 107, 023111.

24 L. Gong, J. Lu and Z. Ye, Transparent conductive Ga-doped $\mathrm{ZnO} / \mathrm{Cu}$ multilayers prepared on polymer substrates at room temperature, Sol. Energy Mater. Sol. Cells, 2011, 95, 1826-1830.

25 J. A. Jeong, Y. S. Park and H. K. Kim, Comparison of electrical, optical, structural, and interface properties of IZO-Ag-IZO and IZO-Au-IZO multilayer electrodes for organic photovoltaics, J. Appl. Phys., 2010, 107, 023111023113.

26 D. R. Sahu and J. L. Huang, Dependence of film thickness on the electrical and optical properties of $\mathrm{ZnO}-\mathrm{Cu}-\mathrm{ZnO}$ multilayers, Appl. Surf. Sci., 2006, 253, 915-918.

27 Y. S. Jung, Y. S. Park, K. H. Kim and W. J. Lee, Properties of AZO/Ag/AZO multilayer thin film deposited on polyethersulfone substrate, Transactions on Electrical and Electronic Materials, 2013, 14, 9-11.

28 J. H. Park, Y. Y. Choi, H. K. Kim and H. H. Lee, The effects of rapid thermal annealing on the electrical, optical, and structural properties of $\mathrm{Nb}^{-\mathrm{TiO}_{2}}$ multilayer electrodes with an inserted nanoscale $\mathrm{Ag}$ layer for organic solar cells, $J$. Appl. Phys., 2010, 108, 083509-083517.

29 Y. Y. Choi, K. H. Choi, H. Lee, H. Lee, J. W. Kang and H. K. Kim, Nano-sized Ag-inserted amorphous $\mathrm{ZnSnO}_{3}$ multilayer electrodes for cost-efficient inverted organic solar cells, Sol. Energy Mater. Sol. Cells, 2011, 95, 1615-1623. 30 J. C. Fan, F. J. Bachner, G. H. Foley and P. M. Zavracky, Transparent heat-mirror films of $\mathrm{TiO}_{2} / \mathrm{Ag} / \mathrm{TiO}_{2}$ for solar energy collection and radiation insulation, Appl. Phys. Lett., 1974, 25, 693-695.

31 G. Leftheriotis, P. Yianoulis and D. Patrikios, Deposition and optical properties of optimized $\mathrm{ZnS} / \mathrm{Ag} / \mathrm{ZnS}$ thin films for energy saving, Thin Solid Films, 1997, 306, 92-99.

32 A. Axelevitch, B. Gorenstein and G. Golan, Investigation of optical transmission in thin metal films, Phys. Procedia, 2012, 32, 1-13.

33 Y. S. Park and H. K. Kim, Flexible indium zinc oxide/Ag/ indium zinc oxide multilayer electrode grown on polyethersulfone substrate by cost-efficient roll-to-roll sputtering for flexible organic photovoltaics, J. Vac. Sci. Technol., A, 2010, 28, 41.

34 K. H. Choi, H. J. Nam, J. A. Jeong, S. W. Cho, H. K. Kim, J. W. Kang, D. G. Kim and W. J. Cho, Highly flexible and transparent $\mathrm{InZnSnO}_{x} / \mathrm{Ag} / \mathrm{InZnSnO}_{x}$ multilayer electrode for flexible organic light emitting diodes, Appl. Phys. Lett., 2008, 92, 223302-223305.

35 J. A. Jeong, H. K. Kim and M. S. Yi, Effect of Ag interlayer on the optical and passivation properties of flexible and transparent $\mathrm{Al}_{2} \mathrm{O}_{3} / \mathrm{Ag} / \mathrm{Al}_{2} \mathrm{O}_{3}$ multilayer, Appl. Phys. Lett., 2008, 93, 0333010-0333012.

$36 \mathrm{~J}$. A. Jeong and H. K. Kim, Low resistance and highly transparent ITO-Ag-ITO multilayer electrode using surface plasmon resonance of $\mathrm{Ag}$ layer for bulk-hetero junction organic solar cells, Sol. Energy Mater. Sol. Cells, 2009, 93, 1801-1809.

37 Y. H. Shin, C. K. Cho and H. K. Kim, Resistance and transparency tunable Ag-inserted transparent InZnO films for capacitive touch screen panels, Thin Solid Films, 2013, 548, 641-645.

38 H. S. Chin, L. S. Chao and C. C. Wu, Crystal, optical, and electrical characteristics of transparent conducting gallium-doped zinc oxide films deposited on flexible polyethylene naphthalate substrates using radio frequency magnetron sputtering, Mater. Res. Bull., 2016, 79, 90-96.

39 A. Klöppel, W. Kriegseis, B. K. Meyer, A. Scharmann, C. Daube, J. Stollenwerk and J. Trube, Dependence of the electrical and optical behaviour of ITO-silver-ITO multilayers on the silver properties, Thin Solid Films, 2000, 365, 139-146.

40 A. G. Milnes and D. L. Feucht, Hetero-Junction and MetalSemiconductor Junctions, Academic, New York, NY, USA, 1972.

41 K. Sugiyama, H. Ishii, Y. Ouchi and K. Seki, Dependence of Indium-Tin-Oxide Work Function on Surface Cleaning Method as Studied by Ultraviolet and X-Ray Photoemission Spectroscopies, J. Appl. Phys., 2000, 87, 295.

42 H. Han, N. D. Theodore and T. L. Alford, Improved conductivity and mechanism of carrier transport in zinc oxide with embedded silver layer, J. Appl. Phys., 2008, 103, 013708-013710.

43 G. Haacke, New figure of merit for transparent conductors, J. Appl. Phys., 1976, 47, 4086-4089. 
44 W. G. Driscoll and W. Vaughan, Handbook of Optics, McGraw-Hill, USA, 1978.

45 L. Kerkache, A. Layadi, E. Dogheche and D. Remiens, Physical properties of RF sputtered ITO thin films and annealing effect, J. Phys. D: Appl. Phys., 2006, 39, 184.

46 H. J. Choi, S. G. Yoon, J. H. Lee and J. Y. Lee, Crystallized Indium-Tin Oxide (ITO) Thin Films Grown at Low Temperature onto Flexible Polymer Substrates, ECS J. Solid State Sci. Technol., 2012, 1, Q106-Q109.

47 A. M. Bazargan, F. Sharif, S. Mazinani and N. Naderi, A high quality ITO/PET electrode for flexible and transparent optoelectronic devices, J. Mater. Sci.: Mater. Electron., 2017, 28, 2962-2969.

48 Y. Wen, H. Liu, S. Yang and L. Fan, Transparent and conductive indium tin oxide/polyimide films prepared by high-temperature radio-frequency magnetron sputtering, $J$. Appl. Polym. Sci., 2015, 132, 42753-42763.

49 T. H. Kim, S. H. Park, D. H. Kim, Y. C. Nah and H. K. Kim, Roll-to-roll sputtered ITO/Ag/ITO multilayers for highly transparent and flexible electrochromic applications, Sol. Energy Mater. Sol. Cells, 2017, 160, 203-210.

$50 \mathrm{~W} . \mathrm{Xu}, \mathrm{Q} . \mathrm{Xu}, \mathrm{Q}$. Huang, R. Tan, W. Shen and W. Song, Fabrication of Flexible Transparent Conductive Films with Silver Nanowire by Vacuum Filtration and PET Mold Transfer, J. Mater. Sci. Technol., 2016, 32, 158-161.

51 T. He, A. Xie, D. H. Reneker, Y. Zhu and A. Tough, HighPerformance Transparent Electrode from a Scalable and Transfer-Free Method, ACS Nano, 2014, 8, 4782-4789.

52 S. Bae, H. Kim, Y. Lee, X. Xu, J. S. Park, Y. Zheng, J. Balakrishnan, T. Lei, H. R. Kim, Y. I. Song, Y. J. Kim, K. S. Kim, B. O. zyilmaz, J. H. Ahn, B. H. Hong and S. Iijima, Roll-to-roll production of 30-inch graphene films for transparent electrodes, Nat. Nanotechnol., 2010, 5, 574578.

53 J. H. Yim, Y. S. Kim, K. H. Koh and S. Lee, Fabrication of transparent single wall carbon nanotube films with low sheet resistance, J. Vac. Sci. Technol., B: Microelectron. Nanometer Struct., 2008, 26, 851-855. 